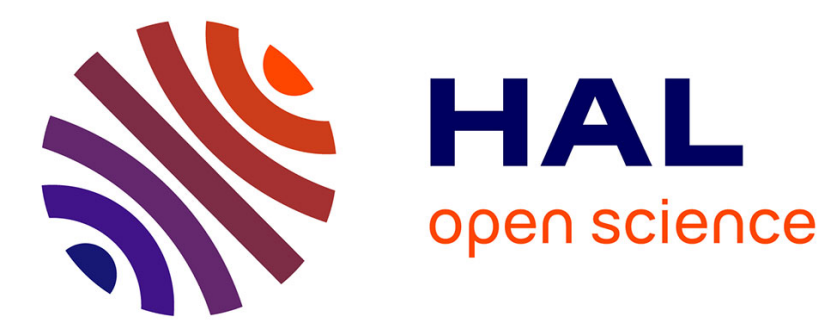

\title{
Évocation et abstraction : Une approche alternative au réalisme des dioramas
}

Eric Triquet

\section{To cite this version:}

Eric Triquet. Évocation et abstraction : Une approche alternative au réalisme des dioramas. Culture et Musées, 2018, L'art du diorama (1700-2000), 32, pp.107-129. 10.4000/culturemusees.2415 . hal03224516

\section{HAL Id: hal-03224516 https://hal.science/hal-03224516}

Submitted on 11 May 2021

HAL is a multi-disciplinary open access archive for the deposit and dissemination of scientific research documents, whether they are published or not. The documents may come from teaching and research institutions in France or abroad, or from public or private research centers.
L'archive ouverte pluridisciplinaire HAL, est destinée au dépôt et à la diffusion de documents scientifiques de niveau recherche, publiés ou non, émanant des établissements d'enseignement et de recherche français ou étrangers, des laboratoires publics ou privés. 


\section{OpenEdition}

Journals

\section{Culture \& Musées}

Muséologie et recherches sur la culture

$32 \mid 2018$

L'art du diorama (1700-2000)

Dossier

\section{Évocation et abstraction : Une approche alternative au réalisme des dioramas}

Evocation and abstraction: an alternative approach to the realism of dioramas

Evocación y abstracción: Un enfoque alternativo al realismo de los dioramas

ÉRIC Triquet

p. $107-129$

https://doi.org/10.4000/culturemusees.2415

\section{Résumés}

Français English Español

Les dioramas sont habituellement associés à une muséographie analogique, fondée sur un principe de similitude. Si ces dispositifs visent à instituer un rapport de transparence avec le réel, d'autres présentations naturalistes vont faire le choix d'une mise en scène abstraite et symbolique. C'est le cas de la galerie «Montagne vivante » du Muséum d'histoire naturelle de Grenoble. À partir de cet exemple, notre article se propose de mettre au jour le fonctionnement sémiotique d'une telle approche muséographique. Il tente de répondre aux trois questions suivantes : cette approche se positionne-t-elle en rupture par rapport aux dioramas ou propose-telle seulement un accès différent au réel ? Si oui, quelle forme de rapport au réel introduit-elle ? Et dans quelle mesure détermine-t-elle de nouvelles modalités de réception du côté du visiteur ? Ce dernier point nous conduit à envisager en conclusion une forme particulière de littéracie, la « littéracie muséale».

Dioramas are usually associated with an analog museography, based on a principle of similarity. If these devices aim to establish a relationship of transparency with reality, other presentations choose to propose abstract and symbolic approach. This is the case of the "Living Mountain" gallery of the Natural History Museum of Grenoble. In support of this example, our publication wishes to reveal the semiotic functioning of this museography. We answer three main questions: does this approach break with the approach of dioramas or does it propose only a different access to reality? So yes which form of relationship to reality does it introduce? And how does it determine new ways of visitor perception? In conclusion, we propose a particular form of literacy, "museum literacy".

Los dioramas suelen asociarse a una museografía analógica, basada en un principio de similitud. $\mathrm{Si}$ estos dispositivos aspiran a establecer una relación de transparencia con lo real, otras 
presentaciones naturalistas hacen la elección de una puesta en escena abstracta y simbólica. Este es el caso de la galería "Montaña viva" del Museo de Historia Natural de Grenoble. A partir de este ejemplo, nuestro artículo propone revelar el funcionamiento semiótico de tal enfoque museográfico. Intenta responder las siguientes tres preguntas: ¿este enfoque rompe con los dioramas u ofrece solo un acceso diferente a la realidad? Si es así, ¿̇qué forma de relación con la realidad introduce? ¿Y hasta qué punto determina nuevas modalidades de recepción del lado del visitante? Este último punto nos lleva a considerar en conclusión, una forma particular de literacia: la "literacia museística".

\section{Entrées d'index}

Mots-clés : diorama, scénographie, sémiotique d'exposition, lecture d'exposition, littéracie muséale, expertise du visiteur

Keywords: diorama, scenography, semiotic exhibition, exhibition reading, museum literacy, visitor expertise

Palabras clave: diorama, escenografía, semiótica de la exposición, lectura de la exposición, lectoescritura museística, experticia del visitante

\section{Notes de la rédaction}

Manuscrit reçu le 7 janvier 2018

Version révisée reçue le 13 juillet 2018

Article accepté pour publication le 3 septembre 2018

\section{Texte intégral}

Du XVIIIe siècle, qui voit la naissance des premiers musées de sciences centrés sur les collections, au XXe siècle, qui voit l'entrée dans l'ère de la communication, les muséums d'histoire naturelle ont connu une évolution que l'on peut qualifier de radicale. La première phase de rupture est le passage de la notion d'objet à mettre en valeur pour lui-même à la notion d' " expôt », d'objet " signifiant » à celui " d'objet illustrant " (Maréchal et al., 2000). Une telle évolution a été possible, selon André Desvallées (1987), dès lors que l'on a accepté l'idée que l'objet de musée n'est pas une fin en soi, mais « le moyen de dire quelque chose ». Après une première période caractérisée par des présentations quasi exhaustives d'objets ordonnés selon les principes de la classification scientifique, voici donc une seconde étape marquée par l'avènement de l'exposition. Des objets sont sélectionnés, associés à d'autres et organisés au regard d'un thème scientifique, d'une idée ou d'une histoire. Ils deviennent « objets prétextes », « objets manipulés » au service d'un discours - celui des concepteurs de l'exposition (Hainard \& Kaehr, 1984). La mise en scène expographique est alors le moyen utilisé par les muséographes pour que ces rassemblements d'objets au sein d'un même espace soient supports de sens. Il s'agit là de la seconde phase de rupture.

C'est dans ce contexte que les dioramas apparaissent et se développent dans les muséums, dès la seconde moitié du XIXe siècle en Amérique du Nord (Tunnicliffe \& Scheersoi, 2015), un peu plus tardivement en France (Pequignot, 2002). Très vite, le terme " diorama » est utilisé pour qualifier tous les procédés muséographiques qui tendent à reconstituer, de façon tridimensionnelle, un environnement naturel. Des animaux naturalisés y sont présentés et mis en situation pour figurer une scène de vie au milieu de décor en fac-similé. Les spécimens ne sont plus présentés selon les critères de la classification systématique ${ }^{1}$, mais replacés dans des décors réalistes et esthétiques rappelant leur milieu naturel. Le diorama, nous dit Bernard Schiele, « joue sur la perception du réel. Il est concret », " épris d'authenticité », " soucieux de réalisme » (Schiele, 1996 : 10). L'enjeu est de provoquer un effet de transparence, une illusion de réalité au musée (Wonders, 1993). Grâce au progrès de la taxidermie, l'animal luimême semble vivant. Il est présenté non plus figé mais dans des attitudes et postures de vie caractéristiques, en interaction avec d'autres animaux et avec son milieu. Les dioramas permettent ainsi de recontextualiser dans un espace muséal des spécimens qui, sans cela, seraient appréhendés indépendamment les uns des autres (Davallon et al., 1992), sans ancrage dans le milieu écologique. Si l'on suit la caractérisation établie par Raymond Montpetit (2007), nous sommes en présence d'une muséographie 
analogique, fondée sur un principe de similitude. Ces installations, nous dit-il, fonctionnent sur le mode de l'image, " en renvoyant à leur situation de référence et affirmant que le vrai est (ou a été) tel hors du musée » (ibid. : 58 ). Ils sont assimilés à des fenêtres sur le monde (Quinn, 2006).

3 Pour parer aux limites induites par cette exigence de réalisme, et aussi pour donner plus de place au désir de créativité des conservateurs, certains d'entre eux vont peu à peu renoncer à l'ambition de représenter fidèlement la nature. Ils misent sur une évocation abstraite de la nature. Leurs présentations s'efforcent de faire " allusion " à une portion de la nature, et non "illusion » (Péquignot, $2002: 40$ ). Les décors sont épurés, débarrassés des éléments " réalistes », lesquels sont remplacés par des objets symboliques, des jeux de lumière et de couleurs qui visent à créer des ambiances naturelles.

4 Le Muséum de Grenoble apparaît comme emblématique de cette orientation². Il nous offre l'opportunité de confronter cette approche muséographique fondée sur l'abstraction et le symbolisme à celle développée par les dioramas réalistes. L'étude présentée est ainsi centrée sur les éléments de mise en scène, supports des effets d'évocation recherchés par le concepteur et son équipe3. Plusieurs questions ont guidé notre réflexion. Ces présentations fondées sur l'abstraction se positionnent-elles en rupture ou proposent-elles seulement un accès différent au réel ? Si oui, quelle forme de rapport au réel introduisent-elles ? Dans quelle mesure déterminent-elles de nouvelles modalités de réception du côté du visiteur ? Enfin, comment redéfinissent-elles la stratégie de communication du musée et l'engagement des concepteurs?

\section{Le cas du Muséum de Grenoble : le choix de l'abstraction}

5 Le Muséum d'histoire naturelle de Grenoble figure en France parmi les premiers muséums de province à procéder à la rénovation complète de ses espaces d'exposition (1986-1989). Si la galerie principale et ses boiseries classées ont été conservées, les vastes armoires vitrées dans lesquelles s'accumulaient les spécimens ont été remplacées par des vitrines présentant des scènes de vie animalière dans un style très épuré. Armand Fayard, conservateur en charge de sa rénovation, concède a posteriori qu'il s'est inscrit « en rupture » avec l'approche classique des dioramas, dans une perspective de « changement radical ", courant le risque que cette dernière soit perçue comme une «provocation » (Fayard, $1997: 12$ ). Il explique sa position en ces termes :
«Outre le fait que je ne suis pas favorable, à notre époque, à donner l'impression que l'on puisse reproduire la nature en vitrine, alors qu'elle est encore présente dans toute sa complexité à l'extérieur, le réalisme des dioramas ne m'a jamais paru propice (mais cela demanderait à être évalué auprès du public) au déclenchement du questionnement d'un visiteur. Pourquoi d'ailleurs le visiteur serait enclin à se poser des questions là où il n'y a aucun mystère ! » (ibid. : 15).

Dans une interview publiée dans La Lettre de l'OCIM lors de l'ouverture du musée sous sa forme rénovée, il livre d'autres éléments d'explication. La reconstitution de l'environnement naturel dans une vitrine ne peut être selon lui que partielle et rarement significative du véritable biotope des espèces. Et d'ajouter « qu’il faut éviter de donner l'impression au visiteur qu'il peut très bien se contenter de voir la nature dans un musée », justifiant du même coup l'absence de diorama au Muséum de Grenoble (id., $1990: 7$ ).

7 À la fois épurée et symbolique, la nouvelle muséographie mise en place par Armand Fayard se distingue radicalement de celle mise en ouvre dans les dioramas anglosaxons (Péquignot, $2002: 40$ ). À propos de la vitrine « Drame en sous-bois », qui met en scène plusieurs relations proies/prédateurs, Armand Fayard précise encore un peu plus son approche muséographique :

« Tout dans cette composition est le reflet de l'esprit dans lequel j’ai développé la

réalisation de la vie des espèces animales en milieu forestier montagnard, 
supprimant tout élément naturel. Il n'y a ni branche, ni roche, ni feuille, ni mousse... rien qui pourrait matérialiser, rendre concret et perceptible directement l'environnement de l'animal. C'est cette absence de repères concrets, connus, c'est l'épuration et l'abstraction qui inquiètent le visiteur adulte » (Fayard 1997 : 13).

L'exigence de réalisme cède la place à l'évocation de la nature au moyen d'un ensemble de marques appelées à fonctionner comme des indices, au sens de Martinet ${ }^{4}$. Le registre de l'iconographie est à l'inverse peu développé : aucun dessin figuratif en arrière-plan ici pour représenter le milieu de vie, seulement des formes à peine ébauchées et des taches de couleurs. La structuration de l'espace répond, enfin, à une organisation très précise, elle est également support de sens. Examinons à cet égard le fonctionnement sémiotique de la galerie principale intitulée " Montagne vivante ", avant de nous intéresser aux vitrines qui la composent.

\section{La galerie principale : l'évocation d'un parcours en milieu montagnard}

9 Cette galerie dédiée à la vie animale en montagne présente des spécimens d'animaux caractéristiques des Alpes, dans des environnements scéniques évoquant leur milieu de vie. Armand Fayard présente par ces mots l'esprit dans lequel il a travaillé pour cet espace.

\footnotetext{
« Le visiteur est invité à un voyage au cœur des Alpes, dans une atmosphère vivante où les jeux de lumière et de couleurs rythment l'espace de microclimats chromatiques. Un voyage pour le beau, le rêve et pourquoi pas un peu d'interrogation » (id., 1994: 27).
}

La succession de vitrines qui structure de part en part l'espace de la galerie principale du musée organise un parcours depuis les étages inférieurs de la montagne (aile droite de la galerie en allant vers le fond) vers les hauts sommets (aile gauche). C'est la notion d'étagement de la vie en montagne qui constitue le fil directeur. Mais aucun parcours imposé ici, ni même de balise pour indiquer le chemin à suivre, seulement des taches de couleurs, des rayons de lumière plus ou moins diffus, des artefacts stylisés, disposés çà et là dans les vitrines par le concepteur5.

11 Du côté droit de la galerie, c'est le vert qui domine, pour évoquer la strate arborée encore très présente dans les basses altitudes. Le contraste est fort avec les couleurs minérales (gris ou blanc) retenues au niveau de l'aile opposée, qui renvoient au substrat rocheux ou à la neige des hauts sommets. La lumière apparaît plus douce et plus tamisée du côté droit, comme filtrée par le couvert végétal, plus intense et plus vive dans les vitrines de gauche. Les artefacts du décor se distinguent quant à eux par leur forme : douce et arrondie dans les vitrines des premiers étages de la montagne ; anguleuse et abrupte du côté opposé pour évoquer les pentes escarpées des hauts sommets. La différenciation fine des milieux de chaque étage fait appel aux mêmes systèmes de marques, mais de façon plus nuancée : taches vertes de teintes différentes pour distinguer la forêt de feuillus de la forêt de conifères, taches bleues pour laisser deviner un coin de ciel et évoquer un milieu ouvert comme la clairière. Enfin, de la même façon, la dimension saisonnière est prise en charge par le registre de la couleur : fond vert ou orangé pour évoquer le printemps ou l'automne, sol blanc pour l'hiver, gris ou marron pour l'automne ou le printemps.

On voit ici s'exprimer le dispositif «technique » de guidage - tel que le qualifie Davallon (1996) - développé au moment de la conception de l'exposition. Celui-ci s'appuie sur un agencement apte à orienter le visiteur à la fois spatialement et conceptuellement. Il passe par une organisation formelle précise, pensée en amont. Cette dernière opère à l'échelle de l'espace global et au niveau de chaque vitrine, où elle contribue à développer des points de vue spécifiques. 


\section{Les vitrines : l'expression de points de vue sur la vie animale en montagne}

Nous retenons pour cette étude seulement deux vitrines du musée : une caractéristique de l'étage montagnard, l'autre de l'étage alpin. Leur analyse permet de comprendre comment le concepteur inscrit sémiotiquement ces vitrines dans le parcours global et comment il développe simultanément une focale sur un aspect de la vie animale.

Figure 1. Salle « Montagne vivante », diorama « Les sangliers »

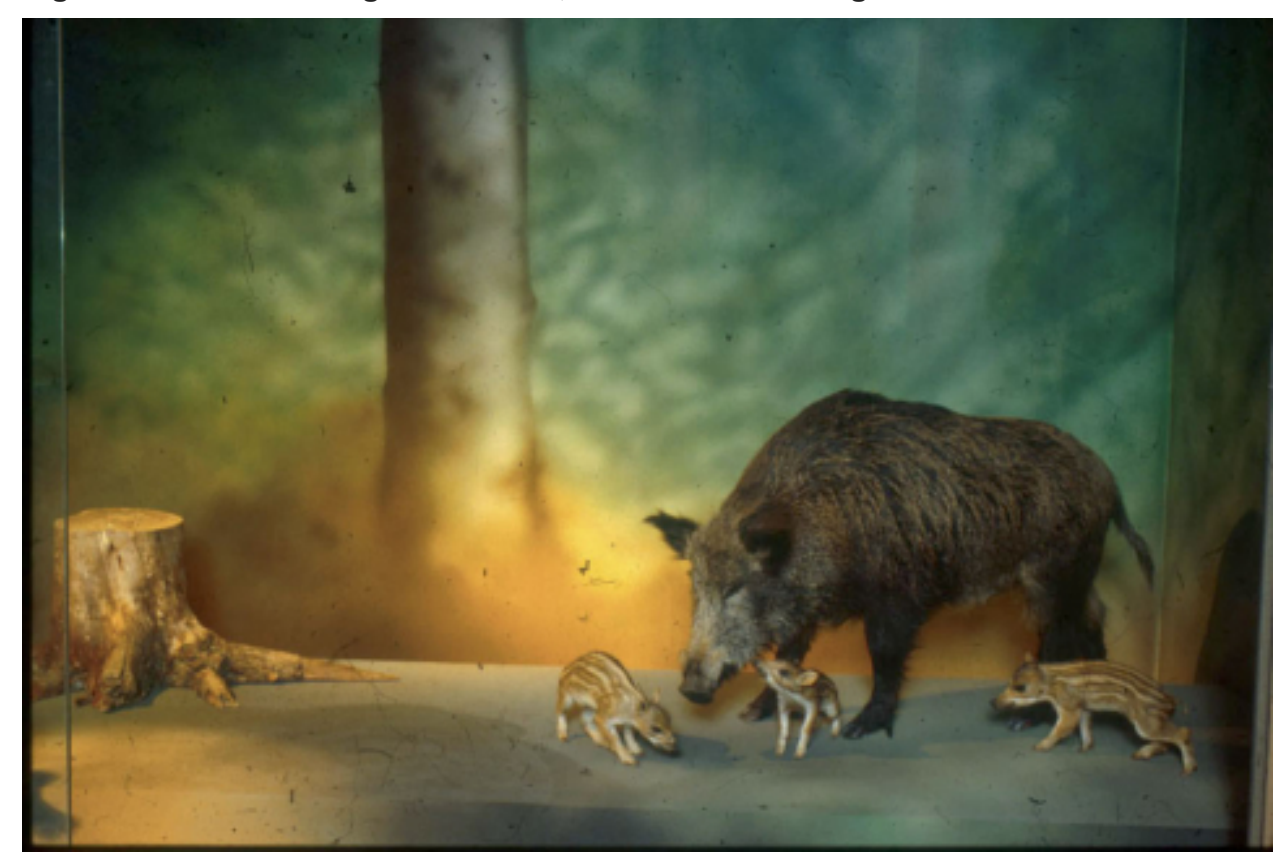

() Muséum d'histoire naturelle de Grenoble

Cette vitrine, localisée à droite de l'allée centrale, fait suite à celle des chevreuils. Plusieurs marques sont disposées pour exprimer le fait que l'on s'enfonce un peu plus profondément dans la forêt : le vert du feuillage est ici continu (indiquant que l'on a quitté la clairière) et la lumière apparaît plus diffuse. Mais d'autres marques tendent à signifier que l'on demeure dans une zone encore facilement accessible à l'homme : rehaussement rectiligne au premier plan symbolisant les bordures d'un chemin, souche d'arbre indifférenciée comme trace de l'activité des forestiers.

La vitrine met en scène une femelle et trois marcassins (regroupés à proximité de la laie). Tous les quatre fouillent le sol avec leur groin, à la recherche de nourriture. Un écureuil est présent, comme dans la vitrine précédente, il constitue le fil directeur de l'évocation de la forêt. Le fond de la vitrine, uniformément vert, entend signifier que la scène se situe au printemps ou en été (période de végétation). Deux autres marqueurs viennent renforcer cette volonté. En premier lieu, la taille des jeunes marcassins, qui naissent à la belle saison. En second lieu, l'absence du mâle, qui ne rejoint le groupe des femelles que le temps de la reproduction, donc plus tôt dans l'année (en décembre). Par la combinaison de ces différents éléments, le concepteur s'efforce de traduire l'idée que le sanglier est étroitement lié au milieu forestier (donc aux premiers étages de la montagne), et de rendre compte de son organisation sociale.

16 La décision de ne pas présenter de mâle dans cette vitrine s’inscrit dans une stratégie délibérée. Elle vise à introduire une focale sur un aspect de cette organisation et susciter un questionnement à son sujet : l'absence du mâle renvoie-t-elle à une absence temporaire ? Si oui, où est-il parti ? Ou bien renvoie-t-elle à une absence plus durable et programmée ? Si c'est le cas, est-ce là un trait caractéristique de l'espèce ? Faut-il en déduire que les mâles ne participent pas à l'éducation des jeunes ? Et enfin, quelle fonction sociale remplit cette séparation (saisonnière) des sexes chez ces animaux?

Figure 2. Salle « Montagne vivante », diorama « Le lagopède alpin et la marmotte » 


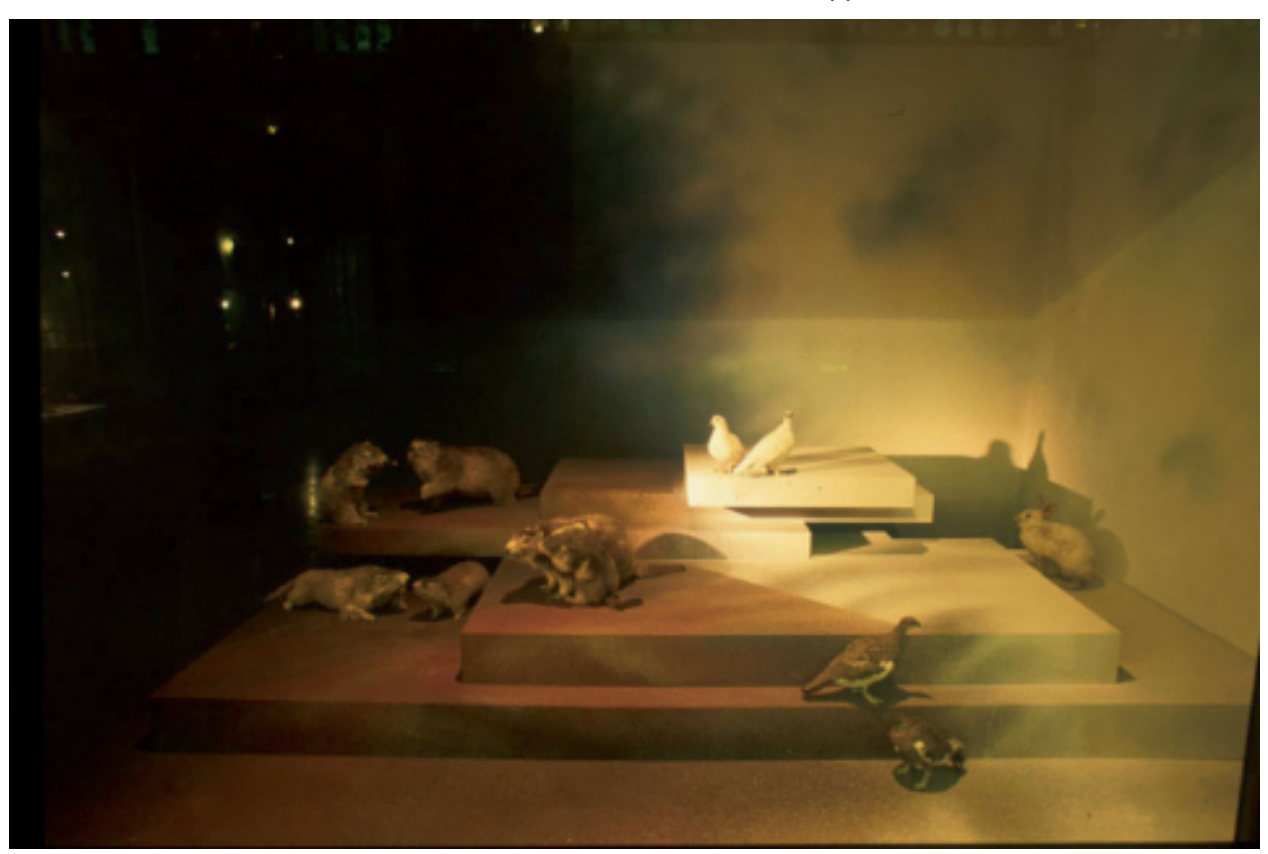

() Muséum d'histoire naturelle de Grenoble

La vitrine retenue est exemplaire de l'approche muséographique retenue. Elle présente trois espèces animales (la marmotte alpine, le lièvre variable et le lagopède alpin) dans leur milieu de prédilection, la prairie des étages subalpin et alpin. La scénographie est marquée par une rupture de teinte au sol - passage de tâches brunâtres sur la partie gauche à un revêtement uniformément blanc à droite - et par une répartition des animaux de part et d'autre de cette limite. Que cherchent à mettre en avant cet agencement particulier et ces jeux de couleurs de la mise en scène ? La partie gauche renvoie au printemps : on y voit des marmottes d'âges différents et deux lagopèdes en livrée gris-marron typique de cette saison. La partie droite, caractérisée par un sol blanc, évoque l'hiver : on retrouve deux lagopèdes, cette fois avec un plumage blanc, accompagnés d'un lièvre variable également blanc, mais plus aucune marmotte pour signifier qu'elles sont en hibernation dans leurs terriers. L'absence de marmottes du côté droit, de même que la couleur blanche des lagopèdes et du lièvre variable, sont à mettre en rapport avec des adaptations de ces animaux à la vie en haute altitude.

L’approche développée au Muséum de Grenoble au début des années 1990 se situe donc bien en rupture avec l'approche analogique et réaliste des dioramas. Il s'agit là de proposer non pas une copie d'un réel présent hors du musée, mais une transposition de ce réel. Du côté de la réception du visiteur, le problème apparaît donc inverse de celui posé par les dioramas : non pas que tout soit transparent, mais au contraire trop opaque, empêchant de décrypter le sens proposé par la mise en scène. Il y a donc une prise de risque au niveau de l'instance de conception qu'il faut pouvoir assumer. On ne peut donc être surpris de voir réapparaitre des éléments réalistes dans les vitrines du Muséum après le départ d'Armand Fayard.

Notre recherche permet de réaliser combien le travail de « lecture " sémiotique de chaque vitrine - en lien avec la lecture scientifique - va être déterminant au moment de la visite. Le visiteur aura en charge non seulement le repérage des différentes marques placées à son intention par le concepteur, mais il devra aussi les confronter entre elles, puis formuler des hypothèses de sens, à la manière d'un enquêteur. Ce travail engage plusieurs opérations que nous proposons de définir ainsi : une inférence qui porte sur les marques formelles de la mise en scène ; une compréhension ou une interprétation visant la construction de significations de second niveau ; et, enfin, une opération d'une autre nature, par laquelle le visiteur engage ses affects et sa sensibilité propres, que nous nommerons "impression ». Nous nous proposons dans la partie qui suit de caractériser cette lecture à partir de ces différentes opérations. 


\section{La lecture de présentations abstraites : entre cognition et expérience sensible}

S'intéresser à la « lecture » de toute présentation muséographique exige de s'intéresser au matériau par lequel l'exposition est en mesure de signifier, donc de produire des effets de sens (Davallon, 1989). Or, face à des présentations abstraites et symboliques, le principe de reconnaissance « à partir d'une situation réelle », comme l'invoque Montpetit (1996) pour les dioramas, apparaît délicat à mettre en ouvre. Par ailleurs, il n'existe aucun code partagé entre concepteurs et visiteurs sur le sens à attribuer aux différents effets de mise en scène. Le sens ne saurait donc être livré tel quel, il demeure à décrypter.

\section{Décrypter les marques de la mise en scène : décodage vs inférence}

Pour comprendre comment néanmoins de telles présentations peuvent signifier, donc produire des effets de sens, Davallon (1996), se référant au théoricien et philosophe du langage Paul Grice, propose de raisonner avec un modèle de communication basé sur le principe d'inférence. Mais pour que les différentes marques placées à l'intention du visiteur soient perçues, encore faut-il que " l'intention informative du concepteur soit suffisamment manifeste », nous dit encore Davallon, ce qui l'amène à caractériser la communication dans l'exposition comme une « communication ostensive inférentielle » (ibid. : 402). Dans le cas des présentations abstraites, ce travail d'inférence doit conduire le visiteur à rendre explicite du sens qui est seulement évoqué, suggéré, comme nous l'avons vu au travers de l'exemple grenoblois. Plus que pour les dioramas, une coopération cognitive du visiteur est nécessaire. Pour s'en convaincre, étudions une des vitrines présentées dans la galerie « Montagne vivante », consacrée aux oiseaux diurnes du milieu forestier.

Figure 3. Salle « Montagne vivante », diorama « les oiseaux diurnes »

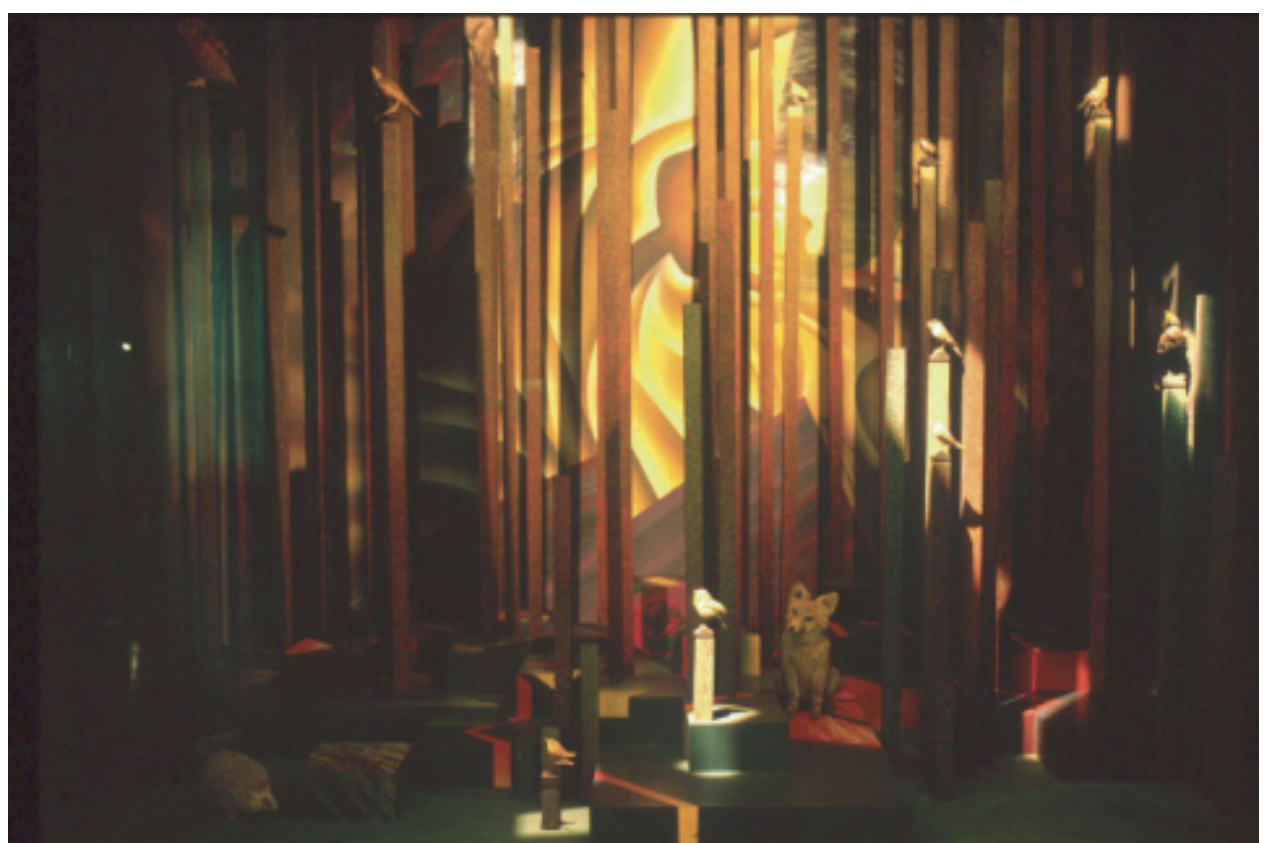

(C) Muséum d'histoire naturelle de Grenoble

Tous les oiseaux sont positionnés sur des pieux rectangulaires de hauteurs différentes et répartis dans tout l'espace de la vitrine. Lorsque le visiteur presse le bouton de la borne placée à proximité, les chants des premiers oiseaux se font entendre, rejoints par d'autres pour atteindre le moment où tous les chants se mêlent alors que la lumière se fait de plus en plus dense ; jusqu'au retour progressif au calme, au fur et à mesure que 
la lumière décline. Que doit comprendre le visiteur de cette mise en scène ? Il lui faut pour commencer assimiler cet ensemble de pieux à une portion de forêt, et les variations de lumière aux variations du jour, de l'aube au crépuscule. Il pourra alors en déduire que certains oiseaux sont matinaux, d'autres plutôt du soir, mais que tous s'associent au final pour créer une " symphonie », selon les mots d'Armand Fayard ${ }^{6}$. Le visiteur doit donc rester en éveil et ouvert à une pluralité de significations. Cette distribution des pieux dans l'espace de la vitrine porte-t-elle du sens ? Et si oui lequel ? Il lui faut pour cela inférer que certains oiseaux vivent en lisière de la forêt (les plus près de la glace), d'autres plus profondément (ceux situés au fond de la vitrine). Et que penser des différences relatives de hauteur de chacun des pieux ? L'inférence est ici plus complexe. Il faut déduire que non seulement les oiseaux ne chantent pas tous au même moment, mais que, en outre ils occupent ce que le naturaliste nomme un " poste de chant ", c'est-à-dire une hauteur préférentielle au niveau de laquelle il se positionne pour chanter.

Le travail de lecture proposé au visiteur débute ainsi par une série d'inférences locales, développées sur chaque élément, puis sur la vitrine dans son ensemble. Elles convoquent des connaissances locales et des compétences « instrumentales » liées à l'inférence. Cependant, ces inférences doivent pouvoir s'inscrire dans l'ensemble du parcours, chacune n'ayant de sens que comparée à d'autres réalisées dans des vitrines proches ou plus lointaines. Cela est manifeste dans le cas des deux vitrines étudiées précédemment. En effet, la caractérisation du milieu et la définition de la saison ne sont ici possibles, comme nous l'avons vu, qu'au travers de nombreuses confrontations et mises en perspective.

Nous nous sommes intéressés jusqu'alors aux opérations qui participent à la construction d'un sens premier, immanent. Elles opèrent sur des signes et correspondent à une saisie et un traitement d'informations en quelque sorte de surface. Mais si l'on considère que le but de toute lecture est la compréhension et l'interprétation, il nous faut à présent aborder une lecture de second niveau.

\section{Construire des sens seconds : comprendre, interpréter}

25 Les études sur la compréhension de textes littéraires ont permis de dégager les caractéristiques principales de ces deux opérations. La plupart d'entre elles, nous semble-t-il, sont transférables à la lecture de l'exposition. En lecture littéraire, la compréhension se définit comme un décryptage au niveau littéral. Il s'agit de retrouver un sens que l'on postule littéral (Jouve, 2001). On admet alors qu'il existe une cohérence intrinsèque au texte. Il en va de même de l'exposition organisée autour d'un topic, ou thème fédérateur. Comprendre renvoie dès lors à une question simple : «Que me dit l'exposition? » Répondre à cette question implique de mettre au jour la logique interne par laquelle chaque objet, chaque élément de la mise en scène trouve sa place et prend du sens. C'est au filtre de ce topic que le visiteur peut apprécier la validité de ses inférences. Il thématise - pourrait-on dire - sa lecture de l'exposition dans un souci d'objectivité.

Dans le cas étudié à Grenoble, c'est le thème de l'étagement du milieu montagnard qui est apparu structurant. Il est tout à la fois le point de focalisation qui donne sens à l'ensemble des inférences et le produit des multiples inférences réalisées par le visiteur. Une fois ce topic appréhendé, il permet au visiteur de se repérer spatialement dans la galerie, en lien avec le monde de référence, celui de l'espace montagnard.

$27 \quad$ Mais l'exposition est marquée, selon Raymond Montpetit, par une « suggestion de sens, par une énonciation plus ou moins claire et entendue, qui enrobe les choses exposées dans une discursivité interprétative » (Montpetit, 1997 : 9). Avec l'interprétation, il s'agit alors d'aller à la recherche d'un sens qui résiste, d'une " illisibilité résiduelle ». Si l'on transpose cette définition, proposée par Yves Reuter (1992) pour le texte littéraire, à l'exposition, l'interprétation répondrait à de nouvelles questions : non plus « que me dit l'exposition ? » mais plutôt « au-delà de ce qu'elle me 
dit, que cherche-t-elle à me dire (qu'elle ne me dit pas explicitement) ? » Que cherche " réellement " à me dire le concepteur par cette façon de disposer les objets, par ces choix formels de mises en scène, etc., dont je n'ai pas encore vu la portée ? Ce qui sousentend qu'elle dit aussi " autre chose », " en plus », différent du sens construit par la compréhension.

À Grenoble, c'est le thème de l'adaptation aux contraintes du milieu montagnard (dont celles liées aux variations saisonnières) qui est traité en arrière-plan tout au long du parcours, et de façon plus marquée au niveau des vitrines situées à gauche. Il s'exprime en cohérence avec le thème de l'étagement des milieux de montagne, lequel, on l'a dit, détermine la structuration de l'espace global de la galerie. Pour accéder à ce niveau de signification le visiteur ne doit pas considérer chaque animal présenté pour lui-même, mais comme un marqueur de cet étagement ${ }^{7}$; et il doit activer le filtre conceptuel de l'adaptation. Si ce n'est pas le cas, il peut tout aussi bien interpréter la différence de couleurs des lagopèdes comme une marque de dimorphisme sexuel, que l'attribuer à des espèces distinctes. Il ne pourra pas non plus relier l'absence de la marmotte côté gauche à l'idée d'hibernation, qui correspond cette fois à une adaptation physiologique et comportementale de cet animal de haute altitude.

Mais le sens premier est parfois si fortement ancré dans la culture du visiteur qu'il lui interdit toute autre façon de le considérer. Aussi, comme le note Davallon, « la relation que le visiteur établit avec l'objet va plus le conduire vers le monde auquel appartient l'objet que vers le monde imaginaire ou conceptuel du concepteur » (Davallon, 1999 : 30). De fait, Jacques Maigret, ancien conservateur en chef au Muséum d'histoire naturelle de Paris, se demande si ce principe de l'allusion - également convoqué lors de la réalisation de la grande galerie de l'Évolution - n'est pas trop complexe et s'il n'est pas nécessaire pour le visiteur de disposer d'un « mode d'emploi » (Maigret \& RaulinCerceau, 2000). C'est là selon nous la principale limite présentée par cette approche sur laquelle nous reviendrons en conclusion.

\section{Investir un imaginaire, une sensibilité propre : l'impression}

30 Exposer, nous rappelle Raymond Montpetit, c'est " réunir des objets et les insérer dans une certaine mise en scène avec le projet que l'ensemble constitue un processus significatif donnant à penser aux visiteurs, mais aussi à rêver, à partir de ce qu'ils perçoivent autour d'eux » (Montpetit, $2003:$ 30). Dès lors, la réception dans l'exposition devient pour le visiteur, dès son entrée, un lieu propice à l'investissement de sa sensibilité, de son imaginaire.

31 L'exposition, dans le cas du Muséum de Grenoble, est un lieu pensé - selon les vœux du conservateur - comme propice à l'expression des émotions. Nous avons rapporté en introduction à la première partie ces propos d'Armand Fayard, concepteur de ces dioramas : " Un voyage pour le beau, le rêve ». Nul besoin d'une étude approfondie pour repérer la recherche d'esthétique ${ }^{8}$ qui a guidé son travail de conception, auquel il a ajouté tout à la fois une tension dramatique et une touche de poésie, pour mieux laisser s'exprimer la sensibilité et l'imaginaire du visiteur. Ces choix déterminent ainsi une nouvelle modalité de réception.

De façon complémentaire à la compréhension et à l'interprétation, Yves Reuter (1992) distingue pour la lecture littéraire une composante qu'il propose d'appeler, faute de mieux, « impression ». Or le sens est fondamentalement effet de sens pour le visiteur qui ressent des émotions et réagit. L'impression pourrait ainsi désigner ces effets produits sur le lecteur - que ceux-ci soient recherchés ou non - et envisagés sur le plan psychoaffectif, donc en lien avec les affects de chaque visiteur, ses goûts, ses sentiments, ses appréhensions. Un espace de liberté est ici ouvert au visiteur. S'il s'en saisit, il pourra s'abstraire provisoirement du guidage mis en place par l'instance de conception, et proposer d'autres recontextualisations, d'autres d'associations qui n’appartiennent qu'à lui. 
33 La forêt, telle qu'elle est mise en scène au Muséum de Grenoble, peut tout aussi bien être perçue comme protectrice ou pleine de dangers, familière ou étrange. Et de la même façon pour les hauts sommets, espaces méconnus, révélés au visiteur par quelques traits insolites de ses occupants - parade nuptiale volée aux mâles tétraslyres, bouquetins surpris en équilibre sur des éperons rocheux -, et qui peuvent nourrir bien des imaginaires. De façon évidente, l'impression, telle que définie, est à considérer comme une composante essentielle de la réception du visiteur dans la mesure où nulle lecture d'exposition ne peut y échapper. Mais il ne saurait être question de considérer cette modalité comme dissociée des trois autres. Elle est appelée, selon nous, à accompagner dès le départ le travail d'inférence, mais encore la compréhension et l'interprétation.

\section{Conclusion}

34 Qu'il s'agisse de dioramas ou de présentations plus abstraites qui empruntent au diorama certaines de ses propriétés, la mise en scène muséographique crée simultanément un espace de perception et un espace de sens selon un équilibre qui varie selon le type de muséographie de référence. Mais dans tous les cas, c'est une réalité reconstruite qui est donnée à voir au visiteur, à laquelle ce dernier accède à partir de ses expériences du monde, sa sensibilité propre, son imaginaire, mais aussi en fonction de son rapport au monde et de ses représentations.

En se présentant comme simple reconstitution de la réalité, le diorama a l'ambition d'instituer un rapport de transparence (Montpetit, 1996). Mais cette idée de transparence demande à être discutée. Michel Van-Praët (1996) rappelle que les dioramas naturalistes sont avant tout des scènes à finalité écologique qui proposent, sous une forme attractive, les conclusions (écologiques, éthiques, etc.) des concepteurs de l'exposition. Or celles-ci ne sauraient être directement accessibles pour l'ensemble des visiteurs. Des connaissances sont exigées. Elles ne relèvent ni de la perception immédiate des choses, ni de la pensée commune - l'une et l'autre pouvant ici fonctionner comme des obstacles, au sens de Bachelard -, mais bien de savoirs scientifiques. Considérons ensuite que l'ambition de pouvoir représenter fidèlement le réel relève d'une forme d'illusion, tant le réel est complexe et multiforme. C'est en fait une image nécessairement figée (Étienne, 2017), partielle et réductrice de la nature qui est proposée aux visiteurs. Elle met en avant une uniformité certes rassurante, mais bien peu conforme à la diversité du monde naturel. Pour toutes ces raisons, les dioramas ne peuvent être pensés comme de simples copies de la nature, mais plutôt comme des faux-semblants. Le recours à la réalité virtuelle ou augmentée, sorte de " digital diorama », fait un pas de plus dans cette direction en offrant au visiteur une expérience immersive dans ce réel reconstruit, présent ou passé. Mais comme dans les dioramas, ces présentations imposent une forme, une image de la nature qui laisse peu d'espace aux visiteurs pour la construction d'autres points de vue.

Avec l'approche abstraite, la représentation du monde apparait plus ouverte et multiforme. Un autre rapport au réel, moins direct, moins immédiat, est installé. Le sens demeure à construire via un travail de lecture exigeant qui requiert, comme nous l'avons vu avec l'exemple grenoblois, non seulement des savoirs mais aussi des compétences de lecture bien spécifiques. Une forme particulière de littéracie est ici en jeu ; nous avons proposé de la nommer « littéracie muséale 9 » (Poli \& Triquet, 2004). Nous l'avons définie comme un ensemble de compétences permettant d'accéder aux multiples niveaux de sens d'une exposition, à partir de l'ensemble des registres sémiotiques mobilisés pour sa conception. Elle renvoie à une lecture experte ${ }^{10} \mathrm{du}$ visiteur, appelée à être mobilisée de façon ciblée et différenciée selon les dispositifs muséographiques.

37 Dès lors, une autre dimension demande à être appréhendée. Elle concerne les phénomènes d'intercompréhension sémantique qui s'opèrent chez le visiteur entre le registre langagier écrit et les autres registres expographiques. Marie-Sylvie Poli (2002) a pu noter que les visiteurs n'attribuaient pas les mêmes effets cognitifs et sémantiques 
au médium pluri-sémiotique que constitue l'exposition et au médium langagier que composent les écrits informatifs. Ce constat est particulièrement intéressant puisqu'il questionne une dimension essentielle de l'expertise du visiteur : la compétence qui permet de lire de concert, en les associant, le non-verbal et le verbal dans l'exposition. Les recherches que nous avons menées en contexte scolaire (Triquet, 2001, 2007) nous ont permis d'examiner de plus près le développement de cette compétence. En proposant cette notion, notre souhait n'était pas d'imposer une nouvelle notion - une de plus ! - mais bien de mettre en avant ses propriétés heuristiques pour penser la réception au musée. Il nous semble alors que dans le contexte actuel de bouleversement des pratiques de médiation muséale, en lien notamment avec le développement du numérique, les concepteurs d'expositions naturalistes gagneront à envisager cette littéracie en amont de toute production, sous peine de courir le risque d'égarer, de déstabiliser, voire de détourner les visiteurs en manque de repères et de références partagées.

\section{Bibliographie}

Davallon (Jean). 1989. « Peut-on parler d'une "langue" de l'exposition scientifique ? », p. 47-59 in Faire voir, faire savoir : la muséologie scientifique au présent / sous la direction de Bernard Schiele. Québec : musée de la Civilisation.

Davallon (Jean). 1996. "À propos de la communication et des stratégies », p. 389-416 in La Science en scène. Paris, Éditions du Palais de la Découverte.

Davallon (Jean). 1999. L'Exposition à l'œuvre. Paris : L'Harmattan (Communication).

Davallon (Jean), Gottesdienner (Hana) \& Poli (Marie-Sylvie). 200o. « Le concept de visiteur expert ». Muséum international, 52(4), p. 60-63.

Davallon (Jean), Grandmont (Gérard) \& Schiele (Bernard). (1992). L'environnement entre au musée. Lyon : PUL : Québec : musée de la Civilisation.

Desvallées (André). 1987. «Un tournant de la muséologie ». Bulletin de recherches sur l'information en sciences économiques, humaines, et sociales, 10, p. 5-12.

Étienne (Noémie). 2017. « La matérialité politique des dioramas », p. 186-193 in Dioramas (catalogue d'exposition, Palais de Tokyo, 14 juin - 10 septembre 2017) / sous la direction de Katarina Dohm, Claire Garnier, Laurent Le Bon et Florence Ostende. Paris : Palais de Tokyo et Flammarion.

Fayard (Armand). 1990. « La microscopie au musée - le Muséum d’histoire naturelle de Grenoble ». La Lettre de l'OCIM, 7, p. 5-9.

Fayard (Armand). 1994. «Le Muséum de Grenoble », p. 11-31 in Muséum d'aujourd'hui. Dijon : OCIM.

Fayard (Armand). 1997. « Histoire d'une mutation ». La Lettre de l’OCIM, 50, p. 11-15.

Hainard (Jacques) \& Kaehr (Roland). 1984. Objets prétextes, objets manipulés. Neuchâtel : musée d'Ethnographie.

Jouve (Vincent). 2001. La Poétique du roman. Paris : Armand Colin.

Maigret (Jacques) \& Raulin-Cerceau (Florence). 2000. " Les collections mises en scène ? », p. 5362 in La Muséologie des sciences et des publics / sous la direction de Jacqueline Eidelman et Michel Van-Praët. Paris : PUF (Éducation et formation).

Maréchal (Jean-Philippe), Grisolia (Sophie) \& Maigret (Jacques) 20oo. « Les objets en exposition : prétextes ou supports de sens ? ", p. 80-89 in Des expositions scientifiques à l'action culturelle, des collections pour quoi faire ? (actes du colloque international de muséologie, Paris, MNHN, 6-7 juillet 1998) / sous la direction de Yves Girault. Paris : Éditions du Muséum national d'histoire naturelle.

Martinet (Jeanne). 1975. Clefs pour la sémiologie. Paris : Seghers.

Montpetit (Raymond). 1996. «Une logique d'exposition populaire : les images de la muséographie analogique ». Publics et Musées, 9, 1er sem., p. 55-103.

DOI : 10.3406/pumus.1996.1071

Montpetit (Raymond). 1997. « Dramaturgie en quatre temps ». La Lettre de l'OCIM, 50, p. 8-10.

Montpetit (Raymond). 2003. «L'exposition, un geste envers les visiteurs ». Médiamorphoses, 9, p. 29-33.

Péquignot (Amandine). 2002. « La taxidermie dans l'histoire des musées d'histoire naturelle ». La Lettre de l'OCIM, hors-série ( Taxidermie »), p. 35-41.

Poli (Marie-Sylvie). 2002. Le Texte au musée : une approche sémiotique. Paris : L’Harmattan. 
Poli (Marie-Sylvie) \& Triquet (Éric). 2004. « De la lecture à l'interprétation de l'exposition de sciences : une approche de la notion de littératie muséale », p. 177-190 in La Littéracie. Conceptions théoriques et pratiques d'enseignement de la lecture-écriture / sous la direction de Christine Barré-de Miniac, Catherine Brissaud et Mariel Rispail. Paris et Montréal : L'Harmattan.

Quinn (Stephen Christopher). 2006. Windows on Nature. The Great Habitat Dioramas of the American Museum of Natural History. New-York : Abrams \& AMNH.

Reuter (Yves). 1992. " Comprendre, interpréter, expliquer des textes en situation scolaire ; à propos d'Angèle ». Pratiques, 76, p. 7-25.

DOI : 10.3406/prati.1992.1675

Schiele (Bernard). 1996. "Introduction ». Publics et Musées, 9 [" Les dioramas »], 1er sem., p. $10-14$.

DOI : $10.3406 /$ comin.1984.1283

Triquet (Éric). 2001. «Écrire et réécrire des textes explicatifs à partir d'une visite au muséum ». Aster, 33, p. 227-253.

DOI : $10.4267 / 2042 / 8782$

Triquet (Éric). 2007. " L'écriture au service du lire dans une exposition de science, la littéracie muséale ». Didaskalia, 30, p. 9-37.

DOI : $10.4267 / 2042 / 23964$

Tunnicliffe (Sue Dale) \& Scheersoi (Annette). 2015. Natural History Dioramas. History, Construction And Educational Role. Dordrechts, Heidelberg, New York et Londres : Springer.

Van-Praët (Michel). 1989. "Contradiction des musées d'histoire naturelle et évolution de leurs expositions ", p. 25-34 in Faire voir, faire savoir : la muséologie scientifique au présent / sous la direction de Bernard Schiele. Québec : musée de la Civilisation.

Van-Praët (Michel). 1996. « Le Muséum national d'histoire naturelle. Témoin de plus de trois siècles et demi d'évolution de la muséologie des sciences ", p. 217-230 in La Science en scène / sous la direction Michèle Brédimas, James Bradburne et Jean-Pierre Roucan. Paris : Presses universitaires de l'École normale supérieure et Palais de la Découverte.

Wonders (Karen). 1993. Habitat Dioramas: Illusion of Wilderness in Museums on Natural History. Uppsala : Almqwist et Wiksell International.

\section{Notes}

1 Pour des raisons muséographiques, mais aussi d'esthétisme et de lisibilité, seulement un petit nombre de spécimens est sélectionné. Cela fait dire à Michel Van-Praët (1989) que la dissociation entre les collections du musée et l'exposition devient radicale.

2 Laquelle s'est également exprimée au tout début des années 1990 à la grande galerie de l'Évolution du Muséum national d'histoire naturelle de Paris.

3 Nous ne prenons donc pas en considération les textes et commentaires audio des bornes placées à proximité des présentations, mais nous avons conscience qu'ils facilitent grandement le travail d'inférence auquel nous allons nous intéresser, comme le montrent plusieurs de nos travaux (Triquet, 2001, 2007).

$4 \mathrm{Au}$ sens où nous l'entendons, l'indice correspond à « ce qui est là, perceptible, manifeste, à la disposition » du visiteur à qui il appartient de "l'identifier pour ce qu'il est, de lui attribuer le sens souhaité » (Martinet, 1975:59).

5 Libre au visiteur de partir par la gauche et de descendre les étages ou de zigzaguer d'un côté à l'autre.

6 Nous reprenons l'expression utilisée lors d'un entretien qu'il nous a accordé en juin 2018.

7 Des études menées au Muséum national d'histoire naturelle dans la grande galerie de l'Évolution ont montré que l'éléphant de « La caravane de la savane africaine » de la grande nef est perçu par de nombreux visiteurs comme un éléphant d'Afrique et non comme un élément du discours sur la biodiversité, pour lequel il est utilisé dans l'exposition (Maréchal et al., 2000).

8 Mais ce jeu sur l'émotion peut être également perçu comme une source de difficulté. Pour Jacques Maigret et Florence Raulin-Cerceau (2000), il est probable que le choc émotionnel provoqué par la mise en scène particulièrement esthétique de la nef de la grande galerie de l'Évolution au Muséum de Paris empêche de percevoir aisément la répartition des spécimens selon leur provenance géographique. Mais les premières études réalisées à l'ouverture du lieu ont montré que ce n'est qu'à partir du moment où il se trouvait débouté dans sa quête de sens que le visiteur semblait se focaliser sur la beauté et l'esthétique du lieu.

9 La littéracie est en fait une notion d'emprunt à laquelle nous avons proposé d'adjoindre l'adjectif "muséale ». La littéracie regroupe au départ un ensemble de pratiques liées à l'écrit mais qui ne se réduisent aucunement à lui. Un élargissement à d'autres champs de compétences (informatique, médias) a pu être proposé dans les définitions récentes. Dans cette perspective, les pratiques de lecture-écriture ne peuvent plus se limiter à des notions comme celles de grammaire, lexique, sémantique, mais doivent prendre en compte des systèmes sémiotiques plus 
larges. Cette extension décisive de la notion nous a ouvert la possibilité d'envisager cette nouvelle forme de littéracie, la « littéracie muséale ».

10 D'autres études ont montré que des visiteurs peuvent même témoigner de la compétence à mémoriser des grammaires de production d'exposition et des discours de commissaires, pour ensuite les insérer dans un processus de compréhension dialectique (Davallon et al., 2000). Pour les auteurs de cette étude, cette compréhension fait du visiteur un « visiteur expert » ou un « visiteur-critique ».

\section{Table des illustrations}

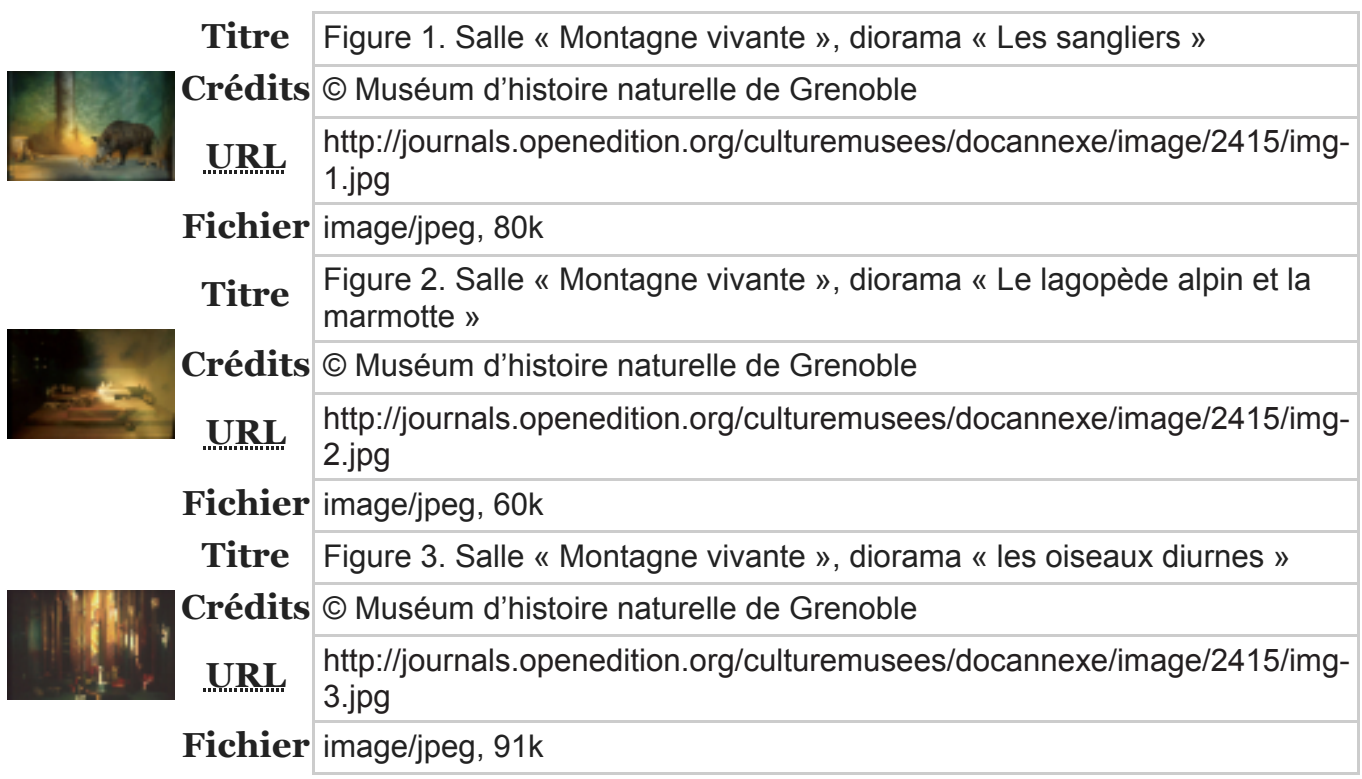

\section{Pour citer cet article}

Référence papier

Éric Triquet, «Évocation et abstraction : Une approche alternative au réalisme des dioramas », Culture \& Musées, 32 | 2018, 107-129.

Référence électronique

Éric Triquet, «Évocation et abstraction : Une approche alternative au réalisme des dioramas », Culture \& Musées [En ligne], 32 | 2018, mis en ligne le 16 janvier 2019, consulté le 11 mai 2021. URL : http://journals.openedition.org/culturemusees/2415; DOI : https://doi.org/10.4000/culturemusees.2415

\section{Auteur}

\section{Éric Triquet}

Avignon Université, Centre Norbert Elias

Éric Triquet est professeur de sciences de l'information et de la communication à l'Avignon Université, et membre du Centre Norbert-Elias (UMR 8562). II est actuellement directeur de la fédération de recherche Agorantic, Culture, Patrimoines, Sociétés numériques (FR-CNRS 3621), et directeur de publication de la revue Culture \& Musées. Après une thèse de doctorat soutenue en 1993 (sous la direction de Jean Davallon) sur la transposition médiatique, il rédige en 2005 une habilitation à diriger des recherches formalisant le concept de littéracie muséale. Ses premières recherches portent sur la relation entre école et musée, et plus précisément sur les dimensions éducatives du musée. Dans le prolongement de son habilitation à diriger des recherches, il s'intéresse aux fonctions du récit dans différents contextes de médiation. II a notamment dirigé le dossier du $\mathrm{n}^{\circ} 18$ de Culture \& Musées, «Le récit dans la médiation des sciences et techniques ». II a mené plusieurs projets de recherche sur cette problématique en prenant comme objet d'étude le musée, les albums de littérature jeunesse, les docu-fictions et les séries télévisées de police scientifique. De façon complémentaire, il a abordé plus récemment le traitement médiatique de controverses scientifiques à partir d'une perspective socio-sémiotique (expositions, presse écrite spécialisée et généraliste). Ses dernières publications sur ce sujet, en collaboration avec Grégoire Molinatti, sont : - 2018. « La controverse à propos du statut phylogénétique de Toumaï : médiatisations et réputations ». Communication \& Langages, 198 ; - « L'encadrement des recherches sur les cellules souches embryonnaires humaines en France : une thématique de choix pour développer une éducation 
aux médias ", in La "Vie » et le "Vivant " : de nouveaux défis à relever dans l'éducation / sous la direction de Michèle Dell'Angelo-Sauvage, Marie-Claude Bernard, Sandrine de Montgolfier et Catherine Simard. Paris : EDP sciences.

Courriel : eric.triquet[at]univ-avignon.fr

Articles du même auteur

Éditorial [Texte intégral]

Paru dans Culture \& Musées, 36 | 2020

Éditorial [Texte intégral]

Paru dans Culture \& Musées, 35 | 2020

Éditorial [Texte intégral]

Paru dans Culture \& Musées, 34 | 2019

Éditorial [Texte intégral]

Paru dans Culture \& Musées, 33 | 2019

Éditorial [Texte intégral]

Paru dans Culture \& Musées, 32 | 2018

Éditorial [Texte intégral]

Paru dans Culture \& Musées, 31 | 2018

Tous les textes...

\section{Droits d'auteur}

Culture \& Musées 